\title{
Downlink Multi-cell Radio Resource Management for Coordinated Base Stations
}

\author{
Olga Muñoz, Eduard Calvo, Josep Vidal, Adrián Agustín \\ Universitat Politècnica de Catalunya, Barcelona, Spain \\ olga.munoz@upc.edu
}

\begin{abstract}
This paper addresses the design of joint multi-cell resource allocation and scheduling with coordination among neighboring base stations (BS). Considering $N_{s}$ neighboring sectors, the solution that maximizes the sum-rate in the covered area is found for TDMA and TDMA/OFDMA access. The optimum solution exhibits low complexity as it presents the useful property that its computation admits a decomposition such that each BS is responsible for the selection of the users to be scheduled in its own sector. This result is proved analytically.
\end{abstract}

Keywords- Coordinated scheduling, multi-cell resource allocation, BS coordination

\section{INTRODUCTION}

In conventional cellular networks, frequency planning is usually considered to keep an acceptable signal-tointerference-plus-noise ratio (SINR) level, especially at cell boundaries. This approach, however, hampers the system spectral efficiency, particularly at low reuse rates. Depending on the allowed complexity and signaling overhead, multi-cell joint inter-cell radio resource allocation may bring significant gains. Intuitively, if users served from different BS experience a low level of interference, radio resources may be reused, thus increasing the system spectral efficiency. On the other side, if the served user experiences large interference, orthogonal transmissions with suitable power allocation are better.

This paper addresses the design of intercell scheduling for neighboring sectors (belonging to different BS) when coordination among these BSs is available, and provides the optimum solution in terms of sum-rate. The weighted sum-rate is also considered in this paper. The weighting factors play the role of implicit linear utility functions, and allow to balance or to prioritize the resource allocation to those users or services with higher weights. In this way fairness issues or QoS criteria can be introduced. When the weighting factors are equal for all the users, maximizing the weighted sum-rate is equivalent to maximizing the system spectral efficiency.

While the solution that maximizes the sum-rate is found to be simple in the TDMA case, it also exhibits reduced complexity for the OFDMA case since it involves the solution of a convex problem with a small number of variables. The solution

This work was supported by the European Union through the project ROCKET ICT-2007-1-215282 and FEDER funds, and by the Spanish/Catalan Science and Technology Commissions through projects 2005SGR-00639, TEC2004-04526, TEC2006-06481/TCM, CONSOLIDER CSD2008-00010 COMONSENS. involves sector-wise independent maximization, which is consistent with maximizing the capacity of each cell through multiuser diversity [1]. Some coordination among the common resources assigned to each neighboring sector is required.

Notation. We use boldface capital font for matrices and boldface lower-case for column vectors. For a matrix $\mathbf{A}$, $A(i, k)$ denotes the $(i, k)$ element of this matrix. $(.)^{T}$ is the transpose operator. $\mathbf{1}_{a \times b}$ denotes an all 1's matrix with dimension $a \times b$ (or vector if $a$ or $b$ are equal to 1 ). $\mathbf{0}_{a \times b}$ is the same but for all zeros vectors. The symbol $\prec$ denotes component-wise inequality for matrices.

\section{TDMA}

We consider frame-based transmission. In our proposal, downlink (DL) transmissions from $N_{s}$ BSs are coordinated. To that end, the DL subframe is further divided in two zones (see Fig. 1):

1) An orthogonal transmission zone, where transmissions from coordinated sectors are orthogonalized. In this way, interference from neighbor (coordinated) sectors is avoided. If TDMA is considered, orthogonality among transmissions from different sectors is granted by allocating them in distinct subtime slots. All the BSs transmit using the whole available bandwidth.

2) A non-orthogonal transmission zone, where radio resources are fully reused by coordinated sectors, and so each user will experience interference from neighboring sectors.

Finally, each BS allocates its own users in a TDMA manner.

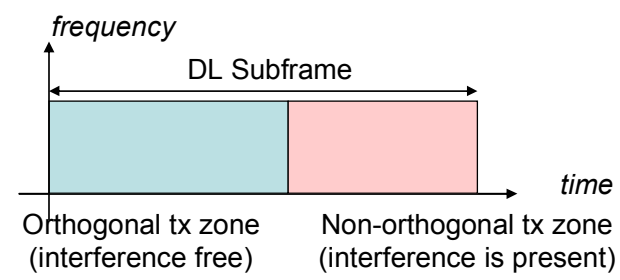

Fig. 1 DL subframe

We will consider in the sequel a multicarrier system and non detailed knowledge of the attenuation per carrier, so that the optimal per-carrier power allocation is uniform. Codewords 
are interleaved and allocated across carriers, and hence the channel capacity may well be approximated by the ergodic [2] (or average) capacity, provided that the number of carriers is sufficiently large and the channel gains are independent.

For the $k$-th user in the $i$-th sector, a lower bound for the ergodic capacity of a SISO direct link transmission is given by [3]:

$$
\tilde{C}_{1}(i, k)=\log \left(1+\frac{\rho P_{B S}}{N_{0} W_{T} L_{i}(i, k)}\right)=\log \left(1+\rho \cdot \operatorname{SNR}_{u}(i, k)\right)
$$

where $P_{B S}$ denotes the power transmitted from the corresponding BS (for simplicity, all the BSs are assumed to transmit with the same power). $L_{i}$ is the pathloss between the $k$-th user in the $i$-th sector to the serving BS. $N_{0}$ is the noise spectral density and $W_{T}$ is the total bandwidth. $S N R_{u}$ is the signal to noise ratio for the considered user. Finally, $\rho$ is a constant subsuming all the impact of the per-carrier fading statistics, and may also include SNR degradation due to imperfect coding. This constant is defined in the Appendix.

In the non-orthogonal mode, simultaneous transmissions from the BS induce interference. A similar lower bound on the ergodic capacity when both the desired user and the interferers occupy the same frequency band is [see Appendix]

$$
\tilde{C}_{2}(i, k)=\log \left(1+\frac{\rho \cdot S N R_{u}(i, k)}{1+S N R_{I}(i, k)}\right)
$$

where

$$
S N R_{I}(i, k)=\sum_{\substack{j=1 \\ j \neq i}}^{N_{s}} \frac{P_{B S}}{N_{0} W_{T} L_{j}(i, k)}
$$

$L_{j}(i, k)$ is the pathloss between $k$-th user in the $i$-th sector and the BS in sector $j$.

Let us denote by $\mathbf{t}$ the vector containing the duration of the the orthogonal zone $t_{1}$ and the non-orthogonal zone $t_{2}$, and by $\mathbf{T}_{1}$ (resp. $\mathbf{T}_{2}$ ) the matrix containing the allocated time in the orthogonal zone (resp. the non-orthogonal zone) for all the users in the coordinated area. The $(i, k)$ element in the matrix corresponds to time allocated to the $k$-th user in the $i$-th sector. The RRM thus needs to optimize $\mathbf{t}, \mathbf{T}_{1}$ and $\mathbf{T}_{2}$.

By considering as the optimization criteria the maximization of the sum of the number of bits/s/Hz that can be reliably delivered in the coordinated area we maximize the spectral efficiency of the system. Instead of $\tilde{C}(i, k)$ we may also consider $C(i, k)=\mu(i, k) \tilde{C}(i, k)$, maximizing therefore the weighted sum-rate of all the users in the coordinated area. The optimization problem can thus be formulated as the following linear program:

$$
\max _{\mathbf{T}_{1}, \mathbf{T}_{2}, \mathbf{t}}\left(\sum_{i=1}^{N_{s}} \sum_{k=1}^{K} T_{1}(i, k) C_{1}(i, k)+\sum_{i=1}^{N_{s}} \sum_{k=1}^{K} T_{2}(i, k) C_{2}(i, k)\right)
$$

$$
\text { s.t. } \quad \mathbf{T}_{1} \succeq \mathbf{0}_{N_{S} \times K}, \mathbf{T}_{2} \succeq \mathbf{0}_{N_{S} \times K}
$$

$$
\begin{aligned}
& \mathbf{t} \succeq \mathbf{0}_{2 \times 1} \\
& \mathbf{1}_{1 \times N_{s}} \mathbf{T}_{1} \mathbf{1}_{K \times 1}-t_{1}=0 \\
& \mathbf{T}_{2} \mathbf{1}_{K \times 1}-t_{2} \mathbf{1}_{K \times 1}=\mathbf{0}_{N_{S} \times 1} \\
& \mathbf{1}_{1 \times 2} \mathbf{t}-1=0
\end{aligned}
$$

A variety of very effective methods to solve a linear program can be found in the literature [4]. However, in this case, the solution may be found analytically and is given next.

Theorem 1. In the orthogonal frame zone, the best user in the whole coordinated area receives all the time resources:

$$
\begin{aligned}
& T_{1}\left(i^{*}, k^{*}\right)=t_{1}, T_{1}(i, k)=0 \text { for }(i, k) \neq\left(i^{*}, k^{*}\right) \\
& \left(i^{*}, k^{*}\right)=\arg \max _{i, k}\left\{C_{1}(i, k)\right\}
\end{aligned}
$$

In the non-orthogonal frame zone, the best user at each coordinated sector is selected (one user per sector). Transmission to these $N_{s}$ users is scheduled simultaneously using all the time resources.

$$
\begin{gathered}
T_{2}\left(i, k_{i}^{*}\right)=t_{2}, T_{2}(i, k)=0 \text { for } k \neq k_{i}^{*} i=1, \ldots, N_{S} \\
k_{i}^{*}=\arg \max _{k} C_{2}(i, k) \text { for } i=1, \ldots, N_{S}
\end{gathered}
$$

For every DL subframe (or each scheduling opportunity) only one zone is selected, i.e. the optimum solution for $t_{1}$ and $t_{2}$ is

$$
\begin{aligned}
& t_{1}=1, t_{2}=0 \text { if } C_{1}^{*}>C_{2}^{*} \\
& t_{1}=0, t_{2}=1 \text { if } C_{1}^{*} \leq C_{2}^{*}
\end{aligned}
$$

with

$C_{1}^{*} \triangleq \max _{i, k} C_{1}(i, k), C_{2}^{*} \triangleq \sum_{i=1}^{N_{s}} C_{2}(i)$ and $C_{2}(i) \triangleq \max _{k} C_{2}(i, k)$

Proof. The utility function in (4) is upper bounded by

$$
\begin{aligned}
\sum_{i=1}^{N_{s}} & \sum_{k=1}^{K} T_{1}(i, k) C_{1}(i, k)+\sum_{i=1}^{N_{s}} \sum_{k=1}^{K} T_{2}(i, k) C_{2}(i, k) \\
& \quad \sum_{i=1}^{(a)} \sum_{k=1}^{N_{s}} T_{1}(i, k) C_{1}^{*}+\sum_{i=1}^{N_{s}} \sum_{k=1}^{K} T_{2}(i, k) C_{2}(i) \\
& =t_{1} C_{1}^{*}+t_{2} \sum_{i=1}^{N_{s}} C_{2}(i) \\
& (c) \\
\quad & t_{1} \max \left(C_{1}^{*}, C_{2}^{*}\right)+t_{2} \max \left(C_{1}^{*}, C_{2}^{*}\right) \stackrel{(d)}{=\max }\left(C_{1}^{*}, C_{2}^{*}\right)
\end{aligned}
$$

where (b) follows from the constraints (7) and (8), and (d) follows from the constraint (9). Note that by selecting $\mathbf{T}_{1}$ according to (10)-(11) and $\mathbf{T}_{2}$ according to (12)-(13), the inequality (a) is achieved with equality. Also by selecting $t_{1}$ and $t_{2}$ according to eq. (14)-(15), the inequality (d) is achieved with equality. Therefore, the maximum of the objective function is given by the last line in equation (17) and this maximum is achieved by the point $\left(\mathbf{T}_{1}^{*}, \mathbf{T}_{2}{ }^{*}, \mathbf{t}^{*}\right)$ in the feasible set described in eq. (10)-(15). 


\section{OFDMA/TDMA}

Next generation wireless systems, such as WiMAX, consider OFDMA at the PHY layer and users are assigned 'slots' which consist on a certain allocation of frequency subchannels for a certain number of symbols. In such a case, an infinite number of possibilities in defining and allocating time-slots arises. Since we target practical solutions to the allocation problem, we shall fix some simplifying structure for the ease of analysis. In particular, and following the philosophy of WiMAX that dynamically allocates data and pilot subcarriers, we consider again two zones for the two different modes of operation: an orthogonal zone and a non-orthogonal zone. In each zone, OFDMA access requires bandwidth and power allocation.

In the orthogonal zone, the users deployed in the coordinated area are orthogonalized in frequency. To that end, the total bandwidth is divided among sectors, with $w_{B S}(i)$ being the bandwidth allocated to the $i$-th sector (it may differ among sectors). Each sector allocates its users within the assigned bandwidth and the BS power is equally split over the assigned bandwith. The number of bits that can be delivered during the orthogonal zone is:

$$
R_{1}(i, k)=t_{1} W_{1}(i, k) \log \left(1+\frac{\rho \cdot P_{B S}}{N_{0} w_{B S}(i) W_{T} L_{s}(i, k)}\right)
$$

In the non-orthogonal zone, frequency resources are reused by all sectors, i.e. every sector allocates its users within the total bandwidth. The number of bits that can be delivered in this zone is therefore:

$$
R_{2}(i, k)=t_{2} W_{2}(i, k) \log \left(1+\frac{\rho \cdot \frac{P_{B S}}{W_{T} L_{s}(i, k)}}{N_{0}+\sum_{\substack{j=1 \\ j \neq i}}^{N_{s}} \frac{P_{B S}}{W_{T} L_{j}(j, k)}}\right)
$$

In this case, the RRM optimizes:

- The duration of each zone

- The bandwidth allocation (normalized to the total bandwidth) for each coordinated sector during the orthogonal zone

- In both zones, the bandwidth allocation (normalized to the total bandwidth) for every user within each sector.

The optimization problem can hence be formulated as follows:

$$
\begin{gathered}
\max _{\mathbf{w}_{1}, \mathbf{w}_{B S}, \mathbf{w}_{2}, \mathrm{t}} \sum_{i=1}^{N_{s}} \sum_{k=1}^{K} \underbrace{t_{1} W_{1}(i, k) \log \left(1+\rho \frac{S N R_{u}(i, k)}{w_{B S}(i)}\right)}_{R_{1}(i, k)}+ \\
\sum_{i=1}^{N_{s}} \sum_{k=1}^{K} \underbrace{t_{2} W_{2}(i, k) \log \left(1+\rho \frac{S N R_{u}(i, k)}{1+S N R_{I}(i, k)}\right)}_{R_{2}(i, k)} \\
\text { s.t. } \quad \begin{array}{l}
W_{1}(i, k) \geq 0, w_{B S}(i) \geq 0, W_{2}(i, k) \geq 0 \\
t_{1} \geq 0, t_{2} \geq 0 \\
\mathbf{W}_{1} \mathbf{1}_{K \times 1}-\mathbf{w}_{B S}=\mathbf{0}_{N_{s} \times 1}
\end{array}
\end{gathered}
$$

$$
\begin{aligned}
& \mathbf{1}_{1 \times N_{s}} \mathbf{w}_{B S}-1=0 \\
& \mathbf{W}_{2} \mathbf{1}_{K \times 1}-\mathbf{1}_{N_{s} \times 1}=\mathbf{0}_{N_{s} \times 1} \\
& t_{1}+t_{2}-1=0
\end{aligned}
$$

The optimum solution to this problem is given by:

Theorem 2. At each DL subframe, only one zone is selected. If the orthogonal mode is selected, the best user (without interference) at each sector is scheduled and the total bandwidth is optimally split among the $N_{s}$ selected users. In the non-orthogonal mode, the best users (considering interference) at each sector transmit simultaneously.

Proof. The proof is based in the key fact that a problem can be optimized by first optimizing over some variables and then over the remaining ones $[4,5]$ :

$$
\max _{\mathbf{x}, \mathbf{y}} f(\mathbf{x}, \mathbf{y})=\max _{\mathbf{x}} \max _{\mathbf{y}} f(\mathbf{x}, \mathbf{y})
$$

First at all, let us define as in eq. (16)

$$
C_{2}(i) \triangleq \max _{k} \log \left(1+\rho \frac{S N R_{u}(i, k)}{1+S N R_{I}(i, k)}\right), C_{2}^{*} \triangleq \sum_{i=1}^{N_{s}} C_{2}(i)
$$

Note that the second term in equation (20) is upper bounded by

$$
\sum_{i=1}^{N_{s}} \sum_{k=1}^{K} R_{2}(i, k) \leq t_{2} \sum_{i=1}^{N_{S}}\left(\sum_{k=1}^{K} W_{2}(i, k)\right) C_{2}(i)=t_{2} \sum_{i=1}^{N_{S}} C_{2}(i)
$$

where equality comes from the constraint (25) and the inequality is achieved with equality by setting

$$
W_{2}\left(i, k_{i}^{*}\right)=1, W_{2}(i, k)=0 \text { for } k \neq k_{i}^{*} \quad i=1, \ldots, N_{S}
$$

Therefore, and using also eq. (27), we can rewrite our problem as

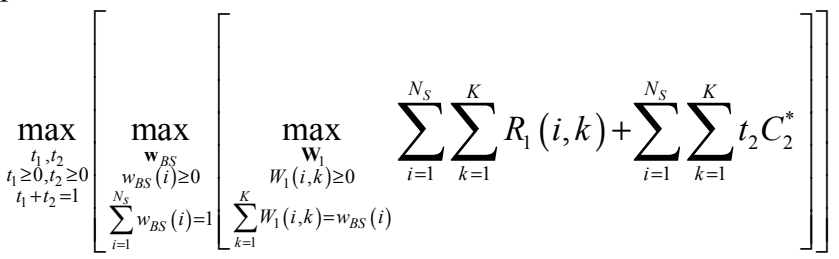

An upper bound of the inner expression can be found by considering

$$
\begin{aligned}
\sum_{i=1}^{N_{S}} \sum_{k=1}^{K} R_{1}(i, k) & \leq t_{1} \sum_{i=1}^{N_{S}}\left(\sum_{k=1}^{K} W_{1}(i, k)\right) \max _{k} \log \left(1+\rho \frac{S N R_{u}(i, k)}{w_{B S}(i)}\right) \\
= & t_{1} \sum_{i=1}^{N_{S}} w_{B S}(i) \log \left(1+\rho \frac{S N R_{u}(i)}{w_{B S}(i)}\right)
\end{aligned}
$$

where equality comes from the constraint (23) and the inequality is achieved with equality by setting

$$
W_{1}\left(i, k_{i}^{*}\right)=w_{B S}(i), W_{1}(i, k)=0 \text { for } k \neq k_{i}^{*} i=1, \ldots, N_{S}
$$

That is, every sector selects the user with the highest $S N R_{u}$ and this user receives the bandwidth assigned to the sector. Therefore, eq. (32) plus eq. (29) provides the maximum of the objective function for any given value of $t_{1}, t_{2}$ and $w_{B S}(i)$ and the problem simplifies to 


$$
\max _{\substack{t_{1}, t_{2} \\ t_{1} \geq 0, t_{2} \geq 0 \\ t_{1}+t_{2}=1}}\left[\max _{\substack{\mathbf{w}_{B S} \\ w_{B S}}} t_{1} \sum_{i=1}^{N_{S}} w_{B S}(i) \log \left(1+\rho \frac{S N R_{u}(i)}{w_{B S}(i)}\right)+t_{2} C_{2}^{*}\right]
$$

For any given value of $t_{1}, t_{2}$ the inner maximization can be performed by solving the following convex problem:

$$
\begin{aligned}
C_{1}^{*}=\max _{w_{B S}(i)} \sum_{i=1}^{N_{S}} w_{B S}(i) \log \left(1+\rho \frac{S N R_{u}(i)}{w_{B S}(i)}\right) \\
\text { s.t. } \quad w_{B S}(i) \geq 0, i=1, \ldots, N_{S} \\
\sum_{i=1}^{N_{S}} w_{B S}(i)=1
\end{aligned}
$$

This problem, with as many variables as sectors under joint coordination, can hence be solved in polynomial complexity by using interior-point methods [6] or standard optimization packages. As the number of sectors is typically moderate, the complexity of the optimization (35)-(37) is small. This renders our approach feasible from a real-time operation standpoint.

Finally, it remains to optimize with respect to $t_{1}, t_{2}$

$$
\begin{array}{ll}
\max _{t_{1}, t_{2}} & t_{1} C_{1}^{*}+t_{2} C_{2}^{*} \\
\text { s.t. } & t_{1} \geq 0, t_{2} \geq 0 \\
& t_{1}+t_{2}=1
\end{array}
$$

Following the same reasoning as in (17)-(c) we find that the optimum is given by eq. (14)-(15)

Differently from the TDMA case, when considering the maximization of the weighted sum-rate in the OFDMA case, it should be jointly obtained the number of users and which ones are served in each sector along with the bandwidth assigned to each sector. Therefore, the optimum solution cannot be derived straightforward from the previous solution. However, the problem can be solved iteratively in this way:

- First, a subproblem is solved within each sector to decide the allocation of resources within the sector

- Second, a common pricing factor, that ensures the common bandwidth constraint, is computed at cluster level and distributed to the coordinated sectors. Using the common pricing factor, the $N_{s}$ sub-problems are solved again until the value of the pricing factor converges.

Due to the lack of space, mathematical details are not included here and are deferred to an upcoming submission.

\section{DISCUSSION AND RESULTS}

It is worth pointing out that in the TDMA case, equation (13) coincides with the conventional opportunistic scheduling where a BS selects the best user at each transmission opportunity and no coordination among BS is considered. On the other hand, equation (11) turns out to be the approach proposed in [7] denoted as coordinated opportunistic scheduling, where the expanded user set is defined as the set of users in the coordinated area. With this approach an expanded multiuser diversity gain is achieved. The approach in [7] presents the drawback of having an efficiency loss due to the fact that only one BS transmits at a given time. The efficiency loss is larger for low interference power.

What Theorem 1 shows is that combining both approaches and selecting one of them depending on the actual level of interference is actually the optimal solution for coordinated scheduling. Note that the user selection within each sector is performed independently. Once the users have been selected, coordination among the sectors is required to decide which transmission mode (orthogonal or non-orthogonal) is activated.

We consider a scenario with 3 coordinated sectors (sectors 1, 2 and 3 in Fig. 2) of radius of $1 \mathrm{~km}$. Users are uniformly distributed within the coordinated area. The BS power is 33 $\mathrm{dBm}$. The noise figure at the MS is $0 \mathrm{~dB}$ and the power spectral density of the noise is $-174 \mathrm{dBm} / \mathrm{Hz}$. The bandwidth is $20 \mathrm{MHz}$. Furthermore, an SNR loss of $4 \mathrm{~dB}$ has been considered to account for the degradation w.r.t. the capacity due to non-ideal modulation and coding. The carrier frequency is $3.55 \mathrm{GHz}$. The antenna gain is $10.6 \mathrm{~dB}$ and the antenna pattern used for each BS sector is specified as [8]

$$
A(\theta)=-\min \left[12\left(\frac{\theta}{\theta_{3 d B}}\right)^{2}, A_{m}\right]
$$

where $A(\theta)$ is the antenna gain in $\mathrm{dB}$ in the direction $\theta, \theta_{3 d B}$ is the $3 \mathrm{~dB}$ bandwidth (corresponding to $70^{\circ}$ ), and $A_{m}=20 \mathrm{~dB}$ is the maximum attenuation.

By considering unitary weights for all the users, i.e $\mu(i, k)=1$, the solution proposed in this paper maximizes the spectral efficiency of the system. Fig. 3 shows the spectral efficiency of the coordinated area, in bits $/ \mathrm{s} / \mathrm{Hz}$, versus the number of coordinated users. Several strategies are compared. First, the conventional opportunistic scheduling (-), where each BS selects the best user for every transmission opportunity. As the BSs transmit in an uncoordinated manner, the users will be interfered by the neighboring BSs. Second, orthogonal scheduling (-+-) where interference is avoided by allowing only one BS to transmit at a given time. Note that, when the number of users in the sector is very high, the set of users with the highest capacities will be close to the BS with high probability and therefore will suffer low interference. Thus, non-orthogonal scheduling is best due to the higher efficiency coming from the fact that all BSs transmit simultaneously. On the other hand, if the number of users is low, the probability that the user with greater capacity perceives low interference decreases. If the perceived interference is not low enough, orthogonal transmission is best as a mean to combat interference. Finally, these strategies are compared to the performance of the proposed approaches in the TDMA (-o-) and OFDMA cases (- $\square-)$. It can be observed that for a low number of users OFDMA outperforms all the previous approaches. For a large number of users, however, the proposed approaches for TDMA and OFDMA offer 
similar performance. The reason is that for a high-number of users non-orthogonal transmission is best and only this mode is activated. Thus, with all the sectors using the entire bandwidth for the corresponding best user, the performance of TDMA and OFDMA is the same.

Note that maximizing the spectral efficiency can sacrifice fairness especially for low mobility situations. However, if the weights are modified to be inversely proportional the traffic served to each user, fairness can be restored. Despite in this case the throughput gains are reduced the trade-off throughput-delay would improve.

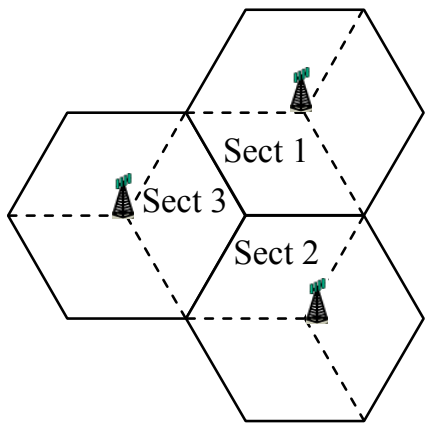

Fig. 2 Example scenario

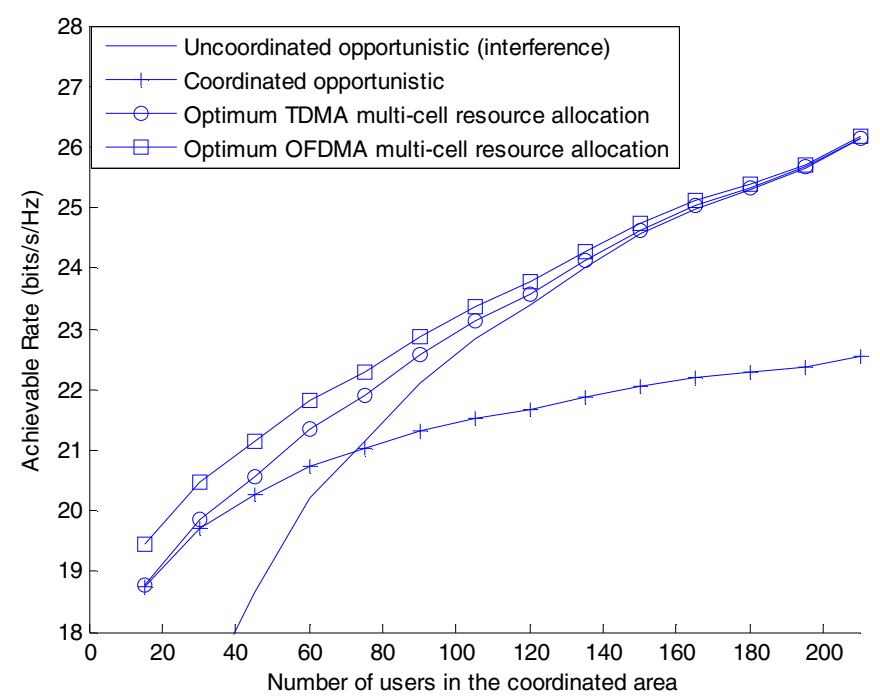

Fig. 3 OFDMA and TDMA comparison

\section{CONCLUSIONS}

In this paper, optimum multi-cell scheduling when coordination among BSs is available has been derived for TDMA and OFDMA. When unitary weights are taken in the weighted sum-rate, simulations have shown significant improvement in spectral efficiency with respect to other approaches available in the literature. The optimum scheme turns out to be very simple and can be decomposed in a form that every BS is responsible from the selection of the users to be scheduled in its own sector. This allows reducing the information to be exchanged among the coordinated BSs.

\section{APPENDIX I}

This appendix considers the effect of simultaneous interferers and derives a lower bound of the ergodic capacity for SISO direct link transmissions when both the desired user and the interferers occupy the same frequency band. Let us first state a bound on the ergodic capacity obtained for simple single user receivers:

$$
\left.R=E\{\log (1+S N R)\}=E\left\{\log \left(1+2^{\log S N R}\right)\right\}\right\}^{(a)} \log \left(1+2^{E\{\log S N R\}}\right)
$$

where inequality $(a)$ comes from the convexity of $\log \left(1+2^{x}\right)$. The SNR is given by:

$$
S N R=\frac{P_{u}\left|h_{u}\right|^{2}}{\sigma^{2}+\sum_{j=1}^{N-1} P_{I, j}\left|h_{i, j}\right|^{2}}=\frac{S N R_{u}\left|h_{u}\right|^{2}}{1+\sum_{j=1}^{N-1} S N R_{I, j}\left|h_{i, j}\right|^{2}}
$$

Replacing (42) in the previous expression:

$$
\begin{aligned}
& R \geq \log \left(1+2^{E\{\log S N R\}}\right)= \\
& =\log \left(1+2^{E\left\{\log S N R_{u}\right\}+E\left\{\log \left|h_{u}\right|^{2}\right\}-E\left\{\log \left(1+\sum_{j=1}^{N-1} S N R_{I, j}\left|h_{i, j}\right|^{2}\right)\right\}}\right) \geq \\
& \stackrel{(a)}{\geq} \log \left(1+S N R_{u} \cdot 2^{E\left\{\log \left|h_{u}\right|^{2}\right\}} 2^{-\log \left(1+\sum_{j=1}^{N-1} S N R_{I, j} E\left\{\left|h_{i, j}\right|^{2}\right\}\right)}\right)= \\
& =\log \left(1+\rho \cdot S N R_{u}\left(1+\sum_{j=1}^{N-1} S N R_{I, j}\right)^{-1}\right)
\end{aligned}
$$

where inequality (a) follows from the concavity of $\log ($.$) , and$ $\rho=2^{E\left\{\log \left|h_{u}\right|^{2}\right\}}$ is a constant that depends on the fading statistics of the channel.

\section{REFERENCES}

[1] R. Knopp and P. Humblet, "Information capacity and powercontrol in single-cell multiuser communications", in Proc. IEEE ICC, June 1995

[2] E. Biglieri, G. Taricco, Transmission and reception with Multiple Antennas: Theoretical Foundations, Foundations and Trends $^{\mathrm{TM}}$ in Communications and Information Theory Series, Now Publishers Inc

[3] E. Calvo, J. Vidal, and J. R. Fonollosa, "Optimal resource allocation in relay-assisted cellular networks with partial CSI", submitted to IEEE Trans. Signal Process., May 2008.

[4] D. P. Bertsekas "Nonlinear Programming". Athena Scientific. $2^{\text {nd }}$ edition, 1999.

[5] D.P. Palomar, M. Chiang, "A Tutorial on Decomposition Methods for Network Utility Maximization", IEEE Journal on Selected Areas in Communications, vol. 24, no. 8, August 2006.

[6] S. Boyd and L. Vandenberghe, Convex Optimization, Cambridge University Press, 2004. Avail. http://www.stanford.edu/ boyd/cvxbook/

[7] W. Choi, J.G. Andrews, "The Capacity Gain from Intercell Scheduling in Multiantenna Systems", IEEE Transactions on Wireless Communications, vol. 7, no. 2, February 2008, pp. 714-725

[8] Gamini Senarath et al, "Multi-hop Relay System Evaluation Methodology (Channel Model and Performance Metric)", IEEE 802.16j$06 / 013$ 